\title{
Efectos a largo plazo del programa PROCONI en preescolares de Tunja (Colombia $)^{5}$
}

\author{
Javier Humberto Parra Pulido \\ Maestrante en Ciencias del comportamiento \\ con orientación en Neurociencias \\ Instituto de Neurociencias, Universidad de Guadalajara \\ Guadalajara, México \\ Correo electrónico: javierhumberto.parra@uptc.edu.co
}

\section{Lucía Carlota Rodríguez Barreto}

Ph.D. Neurociencias Universidad Pedagógica y Tecnológica de Colombia Tunja, Colombia Correo electrónico: lucia.rodriguezb@uptc.edu.co
Recibido: 07/05/2018

Evaluado: $23 / 05 / 2018$

Aceptado: 01/06/2018

\section{Resumen}

Objetivo. Se evaluó el impacto a largo plazo del Programa de Corrección Neuropsicológica Infantil, PROCONI, luego de 3 años de haber sido implementado. Método. Se realizó una investigación prospectiva experimental en una cohorte de 38 niños y niñas de los cuales, el $52.4 \%$ (22) eran de género masculino y $47.6 \%$ (20) femenino. Los participantes tenían edades comprendidas entre 5 y 7 años ( $M=7.12$; $\mathrm{DE}=.96)$, pertenecientes a colegios del área urbana y rural de la ciudad de Tunja. Resultados. En los participantes de la zona urbana y rural se encontraron puntuaciones de neurodesarrollo de acuerdo a la edad y escolaridad, evaluados con el CUMANIN o CUMANES respectivamente y se encontraron correlaciones estadísticamente significativas que oscilaron entre moderadas y fuertes en el desempeño de las evaluaciones de seguimiento en la mayoría de las áreas estimuladas. Discusión. La implementación del programa PROCONI de estimulación neuropsicológica puede tener efectos significativos a largo plazo con el fin de prevenir dificultades de aprendizaje, el fracaso escolar y el ajuste social en las áreas de madurez neuropsicológica.

Estimulación temprana, Programa, Desarrollo Infantil.

5 Para citar este artículo: Parra, J. \& Rodríguez, L. (2019). Efectos a largo plazo del programa PROCONI en preescolares de Tunja (Colombia). Informes Psicológicos, 19(1), pp. 85-104 http://dx.doi.org/10.18566/infpsic.v19n1a05 


\title{
Long-term effects of the PROCONI program in preschool children in Tunja (Colombia)
}

\begin{abstract}
Objective. The long-term impact of the Children's Neuropsychological Correction Program, PROCONI, was evaluated 3 years after it was implemented. Method. An experimental prospective study was carried out in a cohort of 38 boys and girls, of whom $52.4 \%$ (22) were male and $47.6 \%$ (20) female. The participants were between 5 and 7 years old $(M=7.12$, $\mathrm{SD}=.96$ ), belonging to schools in the urban and rural areas of the city of Tunja. Results. In urban and rural participants, it was found that neurodevelopment scores were in accordance with their age and schooling. These scores were evaluated with CUMANIN or CUMANES respectively, and statistically significant correlations were found that ranged from moderate to strong in the performance of follow-up evaluations in most of the areas stimulated. Discussion. The implementation of the PROCONI program of neuropsychological stimulation can have significant long-term effects in order to prevent learning difficulties, school failure and social adjustment in areas of neuropsychological maturity.
\end{abstract}

Keywords Early stimulation, program, child development.

\section{Efeitos a longo prazo do programa PROCONI em crianças em idade pré-escolar em Tunja (Colômbia)}

\section{Resumo}

Objetivo: foi avaliado o impacto a longo prazo do Programa de Correção Neuropsicológica Infantil, PROCONI, 3 anos após sua implementação. Método: um estudo prospetivo experimental foi realizado em uma coorte de 38 meninos e meninas, dos quais $52,4 \%$ (22) eram do sexo masculino e 47,6\% (20) do sexo feminino. Os participantes tinham entre 5 e 7 anos de idade ( $M=7,12, D P=0,96)$, pertencentes a escolas nas áreas urbanas e rurais da cidade de Tunja. Resultados: nos participantes da área urbana e rural acharam-se pontuações de neurodesenvolvimento de acordo com a idade escolaridade, avaliados com o CUMANIN ou CUMANES respetivamente e acharam-se correlações estadisticamente significativas que variavam entre moderadas e fortes no desempenho das avaliações de seguimento na maioria das áreas estimuladas. Discussão: a implementação do programa PROCONI de estimulação neuropsicológica pode ter efeitos significativos a longo prazo com a finalidade de prever dificuldades de aprendizagem, insucesso escolar e lograr 0 ajuste social nas áreas de maturidade neuropsicológica. 


\section{ntroducción}

La madurez neuropsicológica ( $\mathrm{MN}$ ) es considerada como "la respuesta a patrones evolutivos propios del desarrollo normal del individuo, vinculado a la edad cronológica en relación con las funciones cognoscitivas específicas" (Parra, Rodríguez \& Chinome, 2016, p. 127). Su desarrollo está enmarcado por factores multicausales de tipo endógeno y exógeno (Campo, Tuesca \& Campo, 2012; Solovieva \& Quintanar, 2012).

Los factores endógenos incluyen los procesos propios del desarrollo, desde la neurogénsis, la formación del tubo neural, la división encefálica, los procesos de proliferación, migración, diferenciación y apoptosis neuronal y el desarrollo dendrítico que dan lugar a la formación del SNC el cual finaliza su proceso madurativo con la mielinización axonal en las primeras etapas de la adultez (Chávez, 2003; Encalada \& Reino, 2013; Flores \& Ostrosky, 2012; Mendola, Selevan, Grutter \& Rice, 2002; Rosselli, Matute \& Ardila, 2010).

Conjuntamente, la interacción biológico- ambiental pueden potenciar o no el alcance los hitos del desarrollo normal y las habilidades cognitivas (Mora \& Pérez, 2013), donde destacan factores como el nacimiento prematuro (Ríos \& Cano, 2016), el bajo peso al nacer (Parra, Rodríguez \& Chinome, 2015), las condiciones nutricionales (Arias, 2016; Carrasco, Ortiz, Roldán \& Chávez, 2016; Sánchez, 2012), educativas y socioeconómicas (Osorio, Romero, Bonilla \& Aguado, 2016; Portellano, Mateos, Martínez, Granados, \& Tapia, 2009).
Teniendo en cuenta esta interacción biológico-ambiental, la plasticidad cerebral, entendida como las modificaciones a nivel estructural y funcional del SNC, cuyo fin último es adaptarse a los requerimientos del entorno (Muñoz \& Tirapu, 2012; Papalia, Olds \& Feldman, 2009; Parra, 2015; Portellano, 2005), surge como respuesta adaptativa en el desarrollo cognitivo de los niños, donde a la edad de los 3 a 6 años, presenta su etapa más activa y sensible. Por lo tanto, contar con herramientas de estimulación en edades tempranas pueden prevenir la aparición de problemáticas a nivel cognitivo que inciden sobre el desarrollo del ciclo vital del menor.

Al respecto, según cifras de la OMS (2001, citado por Navarro, Meléndez, Sales \& Sancerni, 2012), se calcula que entre el 10 y $20 \%$ de la población infantil presenta algún tipo de trastorno psicológico a nivel mundial, siendo estas cifras similares en países subdesarrollados (Chávez, 2003). En Colombia, la Encuesta Nacional de Salud Mental, para la determinación de la prevalencia y los factores asociados de trastornos contenidos en el DSM IV en niños, estableció que de la población infantil Colombiana, el 3\% presentó algún tipo de trastorno, donde el déficit de atención con hiperactividad y los trastornos de conducta, tuvieron mayores tasas de prevalencia (Gómez et al., 2016). Para los Trastornos específicos del aprendizaje (TEA), no existen cifras concretas en nuestro país, debido a la falta de acceso de algunas poblaciones a servicios especializados de apoyo terapéutico, pedagógico y educación. Sin embargo, algunas aproximaciones como en el trabajo realizado por Benavides, Calvache, Morillo, Ageda y Figueroa (2016), afirman que la prevalencia de los TEA está en un 20\% 
aproximadamente, cifra que coincide con las estadísticas mundiales.

Cabe destacar que los TEA se presentan cuando, en medio de un desarrollo cognoscitivo apropiado, se identifica un área deficitaria en la adquisición de un determinado aprendizaje (Ardila \& Roselii, 2007), con repercusiones futuras en el desenvolvimiento del niño con su medio, la relación con sus pares, su motivación frente al estudio, la adquisición de conocimientos de complejidad cognitiva creciente, abandono escolar y problemas emocionales (Clikeman \& Ellison, 2011; Mora \& Pérez, 2013). Muchos de estos trastornos pasan inadvertidos hasta que la etapa escolar demanda habilidades lectoras, escritoras y de cálculo, convirtiéndose en poblaciones en riesgo (Ministerio de Salud y Protección Social, 2013; Portellano et al., 2009; Urzúa, Ramos, Alday \& Alquinta, 2009).

Esto indica que se debe diseñar e implementar herramientas de prevención e intervención válidas para poblaciones en riesgo de fracaso escolar, siendo esta una tarea pendiente en nuestro medio (Mora \& Pérez, 2013). Se ha calculado que el porcentaje de abandono del colegio en niños o adolescentes con TEA se sitúa alrededor el 40\%, y se relacionan con niveles bajos de motivación, problemas de autoestima, déficit en habilidades sociales y dificultades a nivel laboral o adaptación social a futuro (Rodríguez, Zapata \& Puentes, 2008), problemáticas que pueden haber sido atendidas de forma temprana, mediante programas de estimulación.

A medida que se implementan programas de estimulación a temprana edad en niños, se obtienen mayores beneficios a largo plazo tanto en el desarrollo cognitivo, socialización y éxito académico. Al respecto, Barnett (1995) llevó a cabo una revisión de 36 estudios a largo plazo de estimulación cognitiva en poblaciones con algún tipo de riesgo, evidenciando que la implementación de estos programas puede producir efectos a largo plazo en el Cl, logro académico y ajuste social. Aunque no en todos se encontraron resultados similares, el autor refiere algunas dificultades metodológicas en algunos de ellos.

Por otra parte, uno de los estudios longitudinales más conocidos por su diseño metodológico y seguimiento a diferentes edades de los participantes ha sido el "Abecedarian Project" de Campbell, Ramey, Pungello, Sparling y Jonhson (2010), quienes llevaron a cabo un estudio prospectivo longitudinal para estudiar los beneficios a largo plazo sobre la intervención cognitiva desde edades preescolares, hasta los 21 años. A los participantes se les aplicaron dos programas de intervención, una durante el preescolar y la segunda durante los primeros años escolares. Los resultados ponen de manifiesto que la implementación del programa en edad preescolar se asoció con tamaños de efecto significativo en las habilidades de lectura y matemáticas que persistieron en la edad adulta. La intervención en edades escolares sirvió para mantener los beneficios preescolares para la lectura, pero por sí mismo, los efectos fueron generalmente más débiles que los del programa preescolar.

Otro estudio bien conocido es el "High/ Scope Perry Preschool study" (Belfield et al., 2006), que durante 1962 y 1967 examinaron la vida de 123 afroamericanos nacidos en pobreza y con riesgo de 
fracaso escolar que tenían edades comprendidas entre 3 y 4 años. Los investigadores dividieron los grupos en control y experimental, en donde el primero recibió un Programa preescolar de alta calidad basado en el enfoque de aprendizaje participativo de High/Scope. El efecto del programa fue medido cuando los participantes tenían edad de 40 años. El estudio encontró que los adultos del grupo experimental tenían mejores ingresos, mayores probabilidades de tener un empleo, habían cometido menos crímenes y eran más propensos a graduarse de la escuela secundaria, que los adultos que no habían recibido la intervención.

Baker-Henninhgam y López (2014) realizaron una revisión de programas de estimulación infantil en niños de 0 hasta los 5 años de países en desarrollo. Se realizó una categorización de estudios según el desarrollo infantil mental, motor o de Cl; conducta infantil; escolarización; estado nutricional y salud. Se encontraron 33 trabajos de estimulación temprana, concluyendo que los estudios de intervención benefician el desarrollo mental de los niños y las prácticas de las madres a corto plazo, así como las conductas en entornos sociales y escolares. Revisiones de este tipo a nivel mundial han encontrado resultados similares (véase Maulik \& Darmstadt, 2009; Nores \& Barnett, 2010; Walker et al., 2011).

De estos estudios, cabe resaltar la importancia que tuvo la implementación temprana en edad preescolar, donde la plasticidad cerebral tiene un impacto en el desarrollo de funciones relacionadas al ámbito escolar y social para toda la vida. En Colombia, son pocas las experiencias a largo plazo de intervenciones de este tipo. Una de ellas, las llevadas a cabo por
Pérez y Pollitt (1995 como se citó en Arias, 2016), quienes implementaron un programa combinado de tratamiento nutricional y pedagógico en preescolares desnutridos y de bajo nivel socioeconómico, encontrando beneficios en el grupo experimental. Por su parte, Super, Herrera y Mora (1990) combinaron un programa de compensación nutricional y psicoeducación a padres de familia, con el fin de fortalecer los procesos cognitivos de los niños. Una vez más, los resultados dan cuenta de efectos significativos en los grupos experimentales respecto al control. Attanasio et al., (2014) combinaron la compensación nutricional y estimulación cognitiva; en este trabajo, se identificaron mejoras en el rendimiento cognitivo en el grupo experimental.

Para lo que compete al presente estudio, Rodríguez, Parra y Chinome (2016) llevaron a cabo en el 2014 el diseño, implementación y evaluación del Programa de Corrección Neuropsicológica Infantil PROCONI, que fue aplicado en 76 preescolares del área rural y urbana de la ciudad de Tunja, divididos en grupo control y experimental. Se empleó una metodología cuasi experimental, a partir de un pre test- intervención- post test, utilizando como instrumento de evaluación el Cuestionario de Madurez Neuropsicológica, CUMANIN (Portellano et al., 2009), y el PROCONI como programa de intervención. Este programa, buscó estimular las áreas globales del neurodesarrollo (psicomotricidad, lenguaje, estructuración espacial, visopercepción, memoria visual, atención y ritmo). El programa, fue implementado diariamente en el grupo experimental durante 50 sesiones de 45 minutos cada una. En total se realizaron 200 sesiones durante un periodo de tres meses con cada uno de los grupos de la 
zona rural y urbana. Los resultados evidenciaron diferencias estadísticamente significativas en el grupo experimental respecto al control en las escalas de visopercepción, fluidez verbal, atención y memoria icónica, reportando que el PROCONI podría fortalecer las áreas globales de del neurodesarrollo en los preescolares (véase Chinome, Rodríguez \& Parra, 2017).

En conclusión, la implementación de programas de estimulación cognitiva puede tener efectos significativos a largo plazo con el fin de prevenir dificultades de aprendizaje, el fracaso escolar y el ajuste social. Esto, partiendo desde los principios de estimulación temprana que generan cambios a nivel de neuroplasticidad, consecuencia de la interacción de características genómicas con el medio ambiente, que inducen a cambios positivos a nivel cognitivo en etapas y edades sensibles y críticas del desarrollo (Bonnier, 2008; Knickmeyer, 2008), edades donde se aplicó el PROCONI en la muestra estudiada.

La evaluación a largo plazo de programas de estimulación es limitada y una tarea pendiente en los estudios con este tipo de metodología (Baker-Henninhgam \& López, 2014), por lo tanto, es importante estimar los efectos a largo plazo de este programa debido a que permitirá realizar un seguimiento sobre los perfiles de los grupos, con el fin de identificar su alcance en los procesos de neurodesarrollo y la efectividad a largo plazo. Se plantea como objetivo de este trabajo evaluar el impacto longitudinal del programa PROCONI (Rodríguez, Parra, \& Chinome, 2015) en niños durante un seguimiento de 3 años llevado a cabo en la ciudad de Tunja (Colombia).

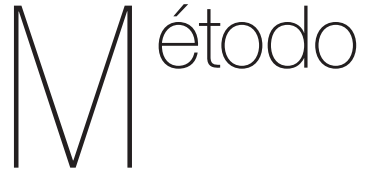

\section{Diseño}

Se realizó una investigación prospectiva experimental en una cohorte de niños escolarizados del área rural y urbana de la ciudad de Tunja (Colombia), que implicó el seguimiento de los participantes 3 años después de la implementación del programa PROCONI (Rodríguez, Parra \& Chinome, 2016) realizada en el año 2014.

\section{Participantes}

La población inicial estuvo conformada por 82 estudiantes de un colegio privado y rural de la ciudad de Tunja de los cuales 38 (46\%) eran de la institución rural y 44 (54\%) de colegio privado. En cada uno de los colegios se realizó un muestreo aleatorio con el fin de seleccionar un grupo control y uno experimental, a este último grupo se le aplicó el programa en el segundo semestre de 2014 en el colegio privado y en el primer semestre de 2015 a los niños del grupo rural. En total fueron 20 participantes del área rural (10 niños y 10 niñas) y 22 (11 niños y 11 niñas) de la urbana, que correspondían a los grupos experimentales de cada una de las instituciones. Los preescolares tenían edades comprendidas entre los 36 a los 78 meses en la aplicación inicial del programa, con una media de edad de 56.81 meses en niños escolarizados en el área rural y de 56.25 meses en los niños del colegio urbano.

La presente investigación contó con la participación de 38 niños/as de zona 
rural y urbana que pertenecían a los grupos experimentales del programa, conformados de la siguiente manera: 21 niños/as del colegio privado (10 niñas y 11 niños) y 16 del colegio rural (10 niñas y 11 niños). La edad media para esta medición fue de 86.82 meses para los niños rurales y 95.23 para los niños urbanos. Como criterios de inclusión se tuvo en cuenta: a) que los niños hubiesen participado en el proyecto de investigación: "Diseño, implementación y evaluación de un Programa de corrección Neuropsicológica en niños de 3 a 6 años de la ciudad de Tunja" (código SGI UPTC 1589); b) que pertenecieran al grupo experimental; c) que contaran con la autorización expresa de participación por medio de la firma del consentimiento informado por parte de los padres y/o representante legal.

Para la presente investigación, no se tomaron en cuenta los participantes del grupo control del estudio original, debido a que al finalizar el estudio base y ver efectos en el programa, se aplicó el programa a los niños del grupo control tal y como lo sugirió el comité de ética de la institución que avaló y financió el proyecto de investigación.

\section{Instrumentos}

Para el desarrollo de este estudio se empleó el Cuestionario de Madurez Neuropsicológica CUMANIN (Portellano et al., 2009), que evalúa el grado de madurez neuropsicológica en niños y niñas de edad escolar comprendida entre los 36 y 78 meses de edad. La prueba constaba de 83 ítems subdivididos en 8 escalas principales y 13 subescalas que se valoran de forma dicotómica. $A$ su vez, la prueba evaluó lateralidad de mano, ojo y pie del niño y clasificándolo en consistente o cruzado. Las escalas principales de la prueba son: Psicomotricidad, Lenguaje articulatorio, Lenguaje comprensivo, Lenguaje expresivo, Estructuración espacial, Viso percepción, Memoria y Ritmo.

La puntuación total (Desarrollo Global), formada por los 83 ítems de las 8 escalas principales, se interpreta en percentiles y, de ésta, se puede obtener un índice en términos de cociente de desarrollo (CD).

La prueba permite además obtener puntuaciones percentiles del Desarrollo Verbal y Desarrollo no-verbal de cada participante. Para este trabajo se utilizó la baremación realizada por Parra \& Rodríguez (2016) para población Boyacense y colombiana. La prueba fue utilizada para evaluar los niños de la muestra con 5 y 6.5 años de edad. En total, fueron evaluados con el CUMANIN 16 participantes (8 niños/as rurales y 8 niños/as de colegio privado).

Además, se empleó el Cuestionario de Madurez Neuropsicológica para Escolares CUMANES (Portellano, Mateos \& Martínez, 2012), que valora el nivel de desarrollo neuropsicológico de los niños y niñas con edades comprendidas entre los 7 a 11 años. Consta de 12 pruebas que se agrupan en 6 secciones evaluando los procesos de lenguaje, visopercepción, función ejecutiva, memoria, lateralidad y ritmo. Debido a que el CUMANES fue diseñado con el propósito de evaluar la madurez neuropsicológica de los niños de edad escolar que no cubría el CUMANIN, esta prueba se empleó para los niños que al momento de 
la aplicación tuvieran edades entre 7 y 8 años. En total fueron evaluados 22 participantes ( 9 niños/as rurales y 13 niños/ as de colegio privado) con el CUMANES, debido a que se encontraban en este rango de edad.

Los instrumentos fueron aplicados debido a que son los únicos que evalúan el constructo de Madurez Neuropsicológica que cuentan con datos normativos para la población colombiana y en específico para población Boyacense -(véase Ávila, 2012; Parra \& Rodríguez, 2016 para CUMANIN y Chinome, Rodríguez \& Pineda, en prensa para CUMANES)-, lo que no afecta la validez interna del estudio en el caso de tomar pruebas no adaptadas para el contexto.

\section{Procedimiento}

El estudio inicial llevado a cabo en el año 2014 de aplicación del PROCONI se puede encontrar publicado en Chinome, Rodríguez y Parra (2017). Se mantuvo contacto con la población con el fin de realizar una evaluación posterior tres años después de la implementación del programa. Para este estudio se tuvieron como fases:

Primera fase: Contacto con las Instituciones Educativas y cronograma de aplicación: Se realizó el contacto pertinente con las instituciones educativas en las cuales se había llevado a cabo el proyecto de investigación previo del área rural y urbana (un colegio de carácter privado y uno público rural de la ciudad de Tunja-Colombia), con el fin de presentar la propuesta de investigación. Una vez aceptada por parte de los organismos encargados, se estableció el contacto con los padres de los participantes a quienes se les brindó toda la información del proyecto. La participación voluntaria en el proyecto quedó evidenciada con la firma del consentimiento informado. Para la aplicación, se realizó un cronograma teniendo en cuenta los tiempos y la utilización de espacios físicos adecuados acordados entre los investigadores y la institución.

Segunda fase: Aplicación y análisis de datos: La aplicación se llevó a cabo de forma individual, en un espacio y tiempo apropiado, en donde solo se encontraba el participante y el investigador o estudiantes del grupo de investigación que participaron en la aplicación, previamente entrenados en áreas del neurodesarrollo infantil y el manejo de las pruebas. Se tuvieron en cuenta las normas técnicas de aplicación del CUMANIN y el CUMANES. Las aplicaciones se realizaron en una sesión por participante en un tiempo que osciló entre los 30 y 45 minutos de aplicación.

Los resultados de las evaluaciones se llevaron a una base de datos en el software SPPSS, versión 23, las cuales recibieron posteriormente un tratamiento estadístico de frecuencias, medias y desviaciones para determinar el rango en el que se ubicaban los participantes fueran bajo, medio o alto. A su vez, se utilizaron correlaciones para identificar la asociación entre el puntaje actual de las pruebas y las diferencias del tratamiento, obtenida por medio de la diferencia matemática de las puntuaciones pre test-post test.

Tercera Fase: Elaboración de Informe y productos entregables: Se realizó el informe pertinente y se dieron resultados a los colegios y padres de 
familia, además de la realización de los productos de divulgación del conocimiento científico propuestos para esta investigación.

\section{Consideraciones Éticas}

El consentimiento informado fue realizado teniendo en cuenta las normas éticas estipuladas en el literal $D$ del artículo 25 de la Ley 1090 del 2006, del código deontológico que regula el ejercicio de la psicología en Colombia, sobre la práctica de la investigación con participantes menores de edad (Ley 1090, 2006). Adicionalmente, se tuvieron en cuenta los lineamientos enmarcados en la Resolución 8430 de 1993 sobre las normas para la investigación en salud en Colombia, calificándose como investigación sin riesgo, ya que no involucró la manipulación de variables biológicas, fisiológicas, sociales o de cambios de conducta intencionado (Resolución 8430, 1993).

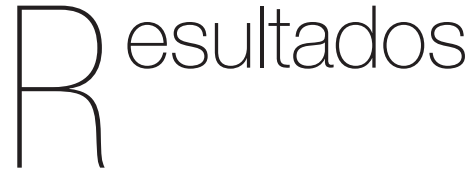

Los resultados de evaluación evidenciaron frecuencias de puntuaciones en la media y por encima de la media en la mayoría de las subescalas en el grupo de niños de colegio privado en las subescalas del CUMANIN (ver Tabla 1). Solo el $12.5 \%$ de la muestra presentó puntuaciones por debajo de la media en las escalas de estructuración espacial y visopercepción. Ningún participante a quien se le aplicó el PROCONI, tuvo resultados en el rango "muy por debajo de la media". En la escala de CUMANES, la frecuencia de puntuaciones, se ubicaron en la media y por encima de la media en la mayoría de las subescalas, evidenciando un índice de madurez neuropsicológica apropiada. En la escala de velocidad lectora el 31\% de los participantes (4 niños/as) presentaron puntuaciones inferiores (decatipo 3).

Tabla 1

Frecuencia de puntuaciones por escala CUMANIN y CUMANES en niños de colegio privado

\begin{tabular}{|c|c|c|c|c|}
\hline \multicolumn{5}{|c|}{ Cuestionario de Madurez Neuropsicológica CUMANIN } \\
\hline & $\begin{array}{l}\text { Muy por debajo } \\
\text { de la media }\end{array}$ & Por debajo de la media & Media & $\begin{array}{l}\text { Por encima de } \\
\text { la media }\end{array}$ \\
\hline Psicomotricidad & $0(0 \%)$ & $0(0 \%)$ & $0(0 \%)$ & $8(100 \%)$ \\
\hline Lenguaje Articulatorio & $0(0 \%)$ & $0(0 \%)$ & $0(0 \%)$ & $8(100 \%)$ \\
\hline Lenguaje Expresivo & $0(0 \%)$ & $0(0 \%)$ & $2(25 \%)$ & $6(75 \%)$ \\
\hline Lenguaje Comprensivo & $0(0 \%)$ & $0(0 \%)$ & $0(0 \%)$ & $8(100 \%)$ \\
\hline Estructuración Espacial & $0(0 \%)$ & $1(12.5 \%)$ & $1(12.5 \%)$ & $6(75 \%)$ \\
\hline Visopercepción & $0(0 \%)$ & $1(12.5 \%)$ & $1(12.5 \%)$ & $6(75 \%)$ \\
\hline Memoria Icónica & $0(0 \%)$ & $0(0 \%)$ & $0(0 \%)$ & $8(100 \%)$ \\
\hline Ritmo & $0(0 \%)$ & $0(0 \%)$ & $0(0 \%)$ & $8(100 \%)$ \\
\hline Fluidez Verbal & $0(0 \%)$ & $0(0 \%)$ & 2 (25\%) & $6(75 \%)$ \\
\hline Atención & $0(0 \%)$ & $2(25 \%)$ & $2(25 \%)$ & $4(50 \%)$ \\
\hline
\end{tabular}




\begin{tabular}{|c|c|c|c|c|}
\hline \multicolumn{5}{|c|}{ Cuestionario de Madurez Neuropsicológica Escolar CUMANES } \\
\hline & $\begin{array}{c}\text { Muy por debajo } \\
\text { de la media }\end{array}$ & Por debajo de la media & Media & $\begin{array}{l}\text { Por encima de } \\
\text { la media }\end{array}$ \\
\hline $\begin{array}{l}\text { Comprensión } \\
\text { Audioverbal }\end{array}$ & $0(0 \%)$ & $0(0 \%)$ & $0(0 \%)$ & $13(100 \%)$ \\
\hline $\begin{array}{l}\text { Comprensión de } \\
\text { Imágenes }\end{array}$ & $0(0 \%)$ & $0(0 \%)$ & $6(46 \%)$ & $7(54 \%)$ \\
\hline Fluidez Fonológica & $0(0 \%)$ & $0(0 \%)$ & $9(69 \%)$ & $4(31 \%)$ \\
\hline Fluidez Semántica & $0(0 \%)$ & $0(0 \%)$ & $7(55 \%)$ & $5(39 \%)$ \\
\hline Comprensión Lectora & $0(0 \%)$ & $0(0 \%)$ & $2(15 \%)$ & $11(85 \%)$ \\
\hline Velocidad Lectora & $0(0 \%)$ & $4(31 \%)$ & $6(46 \%)$ & $3(23 \%)$ \\
\hline Escritura Audiognóstica & $0(0 \%)$ & $0(0 \%)$ & $4(31 \%)$ & $9(69 \%)$ \\
\hline Visopercepción & $0(0 \%)$ & $0(0 \%)$ & $1(13 \%)$ & $12(87 \%)$ \\
\hline $\begin{array}{c}\text { Función } \\
\text { Ejecutiva Tiempo }\end{array}$ & $0(0 \%)$ & $0(0 \%)$ & $9(69 \%)$ & $4(31 \%)$ \\
\hline $\begin{array}{c}\text { Función } \\
\text { Ejecutiva Errores }\end{array}$ & $0(0 \%)$ & $0(0 \%)$ & $1(13 \%)$ & $12(87 \%)$ \\
\hline Memoria Verbal & $0(0 \%)$ & $0(0 \%)$ & $5(38 \%)$ & $8(62 \%)$ \\
\hline Memoria Visual & $0(0 \%)$ & $0(0 \%)$ & $4(31 \%)$ & $9(69 \%)$ \\
\hline Ritmo & $0(0 \%)$ & $0(0 \%)$ & $8(62 \%)$ & $5(38 \%)$ \\
\hline
\end{tabular}

Nota. Fuente: Elaboración Propia. Rangos CUMANIN: Muy por debajo de la media= percentil 1 a 24; Por debajo de la media= percentil 25 a 44; Media= 45 a 64; Por encima de la media= percentil 65 a 99. Rangos CUMANES: Muy por debajo de la media= Decatipo 0 a 2; Por debajo de la media= Decatipo 3 a 4; Media= Decatipo 5 a 6; Por encima de la media= Decatipo 7 a 10.

A su vez, resultados similares se vieron en los niños del grupo rural (ver Tabla 2), en cuanto a que ningún participante presentó puntuaciones en el rango "muy por debajo de la media", evaluados con el CUMANIN o el CUMANES. Sin embargo, se pueden apreciar mayor frecuencia de participantes en el rango de puntuaciones por debajo de la media en las diferentes subescalas respecto al grupo de urbanos.

Tabla 2

Frecuencia de puntuaciones por escala CUMANIN y CUMANES en niños de colegio rural

\begin{tabular}{|c|c|c|c|c|}
\hline \multicolumn{5}{|c|}{ Cuestionario de Madurez Neuropsicológica CUMANIN } \\
\hline & $\begin{array}{c}\text { Muy por debajo } \\
\text { de la media }\end{array}$ & Por debajo de la media & Media & $\begin{array}{l}\text { Por encima de } \\
\text { la media }\end{array}$ \\
\hline Psicomotricidad & $0(0 \%)$ & $0(0 \%)$ & $1(12.5 \%)$ & $7(87.5 \%)$ \\
\hline Lenguaje Articulatorio & $0(0 \%)$ & $0(0 \%)$ & $3(37.5 \%)$ & $5(62.5 \%)$ \\
\hline Lenguaje Expresivo & $0(0 \%)$ & $2(25 \%)$ & $3(37.5 \%)$ & $3(37.5 \%)$ \\
\hline Lenguaje Comprensivo & $0(0 \%)$ & $1(12.5 \%)$ & $1(12.5 \%)$ & $6(75 \%)$ \\
\hline Estructuración Espacial & $0(0 \%)$ & $2(25 \%)$ & $1(12.5 \%)$ & $6(75 \%)$ \\
\hline Visopercepción & $0(0 \%)$ & $1(12.5 \%)$ & $1(12.5 \%)$ & $6(75 \%)$ \\
\hline Memoria Icónica & $0(0 \%)$ & $0(0 \%)$ & $2(25 \%)$ & $6(75 \%)$ \\
\hline Ritmo & $0(0 \%)$ & $1(12.5 \%)$ & $2(25 \%)$ & $5(62.5 \%)$ \\
\hline Fluidez Verbal & $0(0 \%)$ & $0(0 \%)$ & $1(12.5 \%)$ & $7(87.5 \%)$ \\
\hline Atención & $0(0 \%)$ & $4(50 \%)$ & $2(25 \%)$ & $2(25 \%)$ \\
\hline
\end{tabular}




\begin{tabular}{|c|c|c|c|c|}
\hline \multicolumn{5}{|c|}{ Cuestionario de Madurez Neuropsicológica Escolar CUMANES } \\
\hline & $\begin{array}{l}\text { Muy por debajo } \\
\text { de la media }\end{array}$ & Por debajo de la media & Media & $\begin{array}{l}\text { Por encima de } \\
\text { la media }\end{array}$ \\
\hline $\begin{array}{l}\text { Comprensión } \\
\text { Audioverbal }\end{array}$ & $0(0 \%)$ & $0(0 \%)$ & $1(11 \%)$ & $8(89 \%)$ \\
\hline $\begin{array}{l}\text { Comprensión de } \\
\text { Imágenes }\end{array}$ & $0(0 \%)$ & $1(11 \%)$ & $4(45 \%)$ & $4(44 \%)$ \\
\hline Fluidez Fonológica & $0(0 \%)$ & $2(22 \%)$ & $4(45 \%)$ & $3(33 \%)$ \\
\hline Fluidez Semántica & $0(0 \%)$ & $0(0 \%)$ & $6(67 \%)$ & $3(33 \%)$ \\
\hline Comprensión Lectora & $0(0 \%)$ & $0(0 \%)$ & $4(45 \%)$ & $5(55 \%)$ \\
\hline Velocidad Lectora & $0(0 \%)$ & $1(11 \%)$ & $6(67 \%)$ & $2(22 \%)$ \\
\hline Escritura Audiognóstica & $0(0 \%)$ & $1(11 \%)$ & $5(56 \%)$ & $3(33 \%)$ \\
\hline Visopercepción & $0(0 \%)$ & $0(0 \%)$ & $2(22 \%)$ & $7(78 \%)$ \\
\hline $\begin{array}{c}\text { Función Ejecu- } \\
\text { tiva Tiempo }\end{array}$ & $0(0 \%)$ & $2(22 \%)$ & $4(45 \%)$ & $3(33 \%)$ \\
\hline $\begin{array}{l}\text { Función Ejecu- } \\
\text { tiva Errores }\end{array}$ & $0(0 \%)$ & $1(11 \%)$ & $3(33 \%)$ & $5(56 \%)$ \\
\hline Memoria Verbal & $0(0 \%)$ & $1(11 \%)$ & $3(33 \%)$ & $5(56 \%)$ \\
\hline Memoria Visual & $0(0 \%)$ & $1(11 \%)$ & $5(56 \%)$ & $3(33 \%)$ \\
\hline Ritmo & $0(0 \%)$ & $0(0 \%)$ & 7 (78\%) & $2(22 \%)$ \\
\hline
\end{tabular}

Nota. Fuente: Elaboración Propia. Rangos CUMANIN: Muy por debajo de la media= percentil 1 a 24; Por debajo de la media $=$ percentil 25 a 44; Media= 45 a 64; Por encima de la media= percentil 65 a 99. Rangos CUMANES: Muy por debajo de la media= Decatipo 0 a 2; Por debajo de la media= Decatipo 3 a 4; Media= Decatipo 5 a 6; Por encima de la media= Decatipo 7 a 10.

Tabla 3

Relación entre puntajes post test y seguimiento en la escala CUMANIN

\begin{tabular}{ccccc}
\hline & \multicolumn{5}{c}{ Sub Escalas Diferencias CUMANIN } \\
\hline & Rural & & Privado & \\
\hline & Spearman & $\mathrm{p}$ & Spearman & $\mathrm{p}$ \\
\hline Psicomotricidad & .23 & .62 & .14 & .71 \\
Lenguaje Articulatorio & .56 & $.01^{\star *}$ & .9 & $.00^{\star \star}$ \\
Lenguaje Expresivo & .74 & $.05^{\star}$ & .85 & $.04^{\star}$ \\
Lenguaje Comprensivo & .74 & $.05^{\star}$ & .8 & $.03^{\star}$ \\
Estructuración Espacial & .47 & .28 & .3 & .43 \\
Visopercepción & .59 & $.00^{\star \star}$ & .71 & $.00^{\star \star}$ \\
Memoria Icónica & .77 & $.03^{\star}$ & .91 & $.00^{\star \star}$ \\
Ritmo & .26 & .57 & .25 & .52 \\
Fluidez Verbal & .69 & $.05^{\star}$ & .73 & $.02^{\star}$ \\
Atención & .86 & $.01^{* \star}$ & .57 & $.01^{\star \star}$ \\
\hline
\end{tabular}

Nota. Fuente: Elaboración Propia. *Nivel de significancia al nivel 0.05; ** Nivel de Significancia al nivel 0.01 
Para determinar la relación existente entre los resultados del programa de estimulación y el seguimiento actual, se creó una variable de ganancia de puntuaciones, restándole el valor del resultado del post test a las del pre tests del grupo experimental en cada una de las subescalas del CUMANIN. Las variables de diferencia se correlacionaron con su respectiva variable de seguimiento (ver Tabla 3). Por ejemplo, al puntaje de la diferencia entre el pre test y el post test de la escala de psicomotricidad, se correlacionó con la puntuación de psicomotricidad de la evaluación de seguimiento. Este procedimiento se realizó para todos los participantes del grupo rural y urbano a quienes se les aplicó el PROCO$\mathrm{NI}$. Los resultados evidencian relaciones que oscilan entre moderadas y fuertes y son estadísticamente significativas entre las medidas de seguimiento y la ganancia de puntuaciones en la mayoría de las subescalas en ambos grupos (privado y rural).

Para los participantes a quienes se les aplicó el CUMANES también se creó una variable de diferencias entre las puntuaciones pre y post test obtenidas en el CUMANIN, similar a las del análisis anterior (ver Tabla 4). Para este análisis, y entendiendo que en el CUMANES se evalúa MN a mayor edad y con escalas adicionales, se realizaron relaciones entre todas las subescalas del CUMANES y las ganancias del CUMANIN. Al respecto, los resultados muestran relaciones que oscilan entre moderadas y fuertes en diferentes subescalas del CUMANES. La variable de ganancia de ritmo fue la que más se relacionó con escalas del CUMANES. A su vez las escalas de velocidad lectora y escritura audiognóstica, se relacionaron con las ganancias del PROCONI en las escalas de lenguaje comprensivo, psicomotricidad y lenguaje expresivo.

Tabla 4

Relación entre puntajes post test y seguimiento en la escala CUMANES

\begin{tabular}{|c|c|c|c|c|c|c|c|c|}
\hline & $\begin{array}{l}\text { Diferencia Psi- } \\
\text { comotricidad }\end{array}$ & $\begin{array}{l}\text { Diferencia } \\
\text { Lenguaje } \\
\text { Expresivo }\end{array}$ & $\begin{array}{l}\text { Diferencia } \\
\text { Lenguaje } \\
\text { Compren- } \\
\text { sivo }\end{array}$ & $\begin{array}{c}\text { Diferencia } \\
\text { Estructura- } \\
\text { ción Espacial }\end{array}$ & $\begin{array}{l}\text { Diferencia } \\
\text { Viso } \\
\text { percepción }\end{array}$ & $\begin{array}{l}\text { Diferencia } \\
\text { Memoria } \\
\text { Icónica }\end{array}$ & $\begin{array}{l}\text { Diferencia } \\
\text { Ritmo }\end{array}$ & $\begin{array}{c}\text { Diferencia } \\
\text { Fluidez } \\
\text { Verbal }\end{array}$ \\
\hline Fluidez Fonológica & & & & $.43^{*}$ & & $.44^{*}$ & $.60^{\star \star}$ & \\
\hline Fluidez Semántica & & & & & $.46^{\star}$ & & $.61^{\star *}$ & \\
\hline Comprensión Lectora & & & $.52^{*}$ & & & & & \\
\hline Velocidad Lectora & $.48^{*}$ & $.48^{*}$ & & & & & $.59^{* *}$ & \\
\hline Escritura Audiognóstica & & $.57^{* *}$ & & & & & $.49^{*}$ & \\
\hline Visopercepción & & & & & $.83^{\star \star}$ & & & \\
\hline Función Ejecutiva Tiempo & & & & & & & $-.57^{*}$ & $-.52^{*}$ \\
\hline Memoria Verbal & & & & & & & $.49^{*}$ & $.57^{\star \star}$ \\
\hline Memoria Visual & & & & & & $.88^{* *}$ & & \\
\hline Ritmo & & & & & & & $.83^{\star *}$ & \\
\hline
\end{tabular}




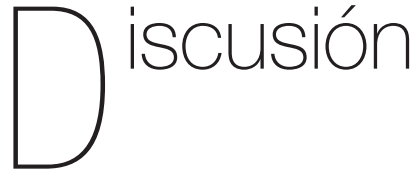

El objetivo del presente estudio fue el de evaluar el impacto longitudinal del programa PROCONI (Rodríguez, Parra \& Chinome, 2015) en niños durante un seguimiento de 3 años. Los resultados obtenidos en la evaluación longitudinal, mostraron puntuaciones normales para los grupos tanto rural como urbano en el desempeño de las pruebas (fueran CUMANIN o CUMANES). Al respecto, ninguno de los participantes, evidenció en los resultados de las escalas, puntuaciones en la categoría "muy por debajo de la media" lo que indicaría que requieren tomar "medidas correctivas" (Portellano et al., 2009, p. 60).

Sin embargo, se presentaron mayores frecuencias de participantes del área rural en el rango "por debajo de la media" que en la urbana. Diversos estudios enmarcan mejores desempeños en tareas neurocognitivas en niños de zona urbana que los de las rurales como en las habilidades visoespaciales (Galindo, Solovieva, Machinskaya \& Quintanar, 2013), tareas cinestésicas, memoria táctil, motricidad, autocontrol y síntesis espaciales (Ortiz \& Añari), entre otras. Estas diferencias a su vez pueden estar asociadas a factores sociales y ambientales que interaccionan con aspectos de maduración cerebral (Arana \& Guisely, 2012; Medina et al., 2015; Mora \& Pérez, 2013).

Por lo tanto, se podría decir como hipótesis que, aunque el PROCONI en primera medida pudo ayudar a estimular áreas implicadas en la madurez neuropsicológica, luego de la aplicación del programa, permanecer inmerso en un ambiente menos enriquecido respecto al grupo de zona urbana, sigue dejando atrás en puntuación a los niños de zona rural.

Se observa por ejemplo que antes de la aplicación del programa, el grupo de niños rurales presentaban mayores frecuencias de puntuaciones por debajo y muy por debajo de la media en todas las escalas (véase tabla 3 estudio de Chinome, Rodríguez \& Parra, 2017), donde las frecuencias mayores de puntuaciones bajas se presentaron para las subescalas de lenguaje expresivo (70\%) y visopercepción (65\%). En el presente estudio se evidenció una frecuencia de puntuaciones por debajo y muy por debajo de la media de dos participantes (25\%) en este rango inferior para lenguaje expresivo y solo un participante (12.5\%) para visopercepción.

Además, aunque algunos de los participantes se encontraron en diferentes subescalas dentro del rango de "por debajo de la media", se debe aclarar que dichas puntuaciones no indican dificultades de tipo patológico y tienden a ajustarse con la estimulación (Portellano, Mateos \& Martínez, 2012). Estudios como el Abecedarian Project (Campbell et al., 2010), a su vez, muestran mayores beneficios en la estimulación a largo plazo cuando es aplicado a edades preescolares, e inclusive repercuten positivamente en factores sociales y ambientales como se pudo observar en el estudio de "High/Scope Perry Preschool study" (Belfield et al., 2006).

Para el presente estudio consideramos que las escalas del PROCONI que mayores resultados tuvieron a largo plazo debido a la estimulación, fueron las áreas de lenguaje expresivo, comprensivo, 
visopercepción y memoria icónica en el grupo de niños evaluados con ambas pruebas; y las de psicomotricidad, estructuración espacial y ritmo para el grupo de niños urbanos y rurales evaluados con el CUMANES. Estos resultados van de la mano con diferencias estadísticamente significativas y tamaños del efecto que oscilaron entre mediano y grande entre la evaluación pre tratamiento y post tratamiento del estudio de pilotaje anterior en ambos grupos poblacionales (véase Chinome, Rodríguez \& Parra, 2017; Rodríguez, Parra \& Chinome, 2016) y que actualmente se conserva el efecto dada las relaciones moderadas y fuertes entre las escalas (véase Tabla 3 y 4), además de presentar mayores rangos de puntuaciones en la media y por encima de la media en dichas escalas para el presente estudio (véase Tabla 2).

El aprovechamiento de la edad preescolar de 3 a 6 años, etapa en la que procesos como la plasticidad cerebral presenta su etapa más activa y sensible (Gutiérrez, Lazarte \& Alarcón, 2016), puede estar asociada con los resultados de las escalas que presentaron puntuaciones en la media y por encima de la media en la mayoría de las subes calas para los niños de zonas urbanas (véase Tabla 1) y en los niños de zonas rurales (véase Tabla 2). Al respecto, las correlaciones llevadas a cabo entre la diferencia de la medida de ganancia con la evaluación actual, identificaron relaciones moderadas y fuertes estadísticamente significativas entre las subescalas, tanto en CUMANIN como en CUMANES, indicando que la implementación del PROCONI puede estar asociado a las buenas puntuaciones presentadas por los grupos. Conjuntamente al programa, la interacción biológico-ambiental puede potenciar o no el alcance los hitos del desarrollo normal y las habilidades cognitivas (Mora \& Pérez, 2013).

A su vez, en la prueba CUMANES, se encontraron relaciones estadísticamente significativas en escalas que miden habilidades superiores como la función ejecutiva, la lectura y escritura (véase Tabla 4), a partir de la estimulación de procesos como estructuración espacial, memoria icónica, visopercepción, lenguaje comprensivo y procesos rítmicos. Diferentes estudios concuerdan en identificar que la estimulación a nivel neuropsicológico, puede jugar un papel predictor en la adquisición de habilidades escolares a largo plazo como en las matemáticas, la lectura y la escritura (Guarneros \& Vega, 2014; Valdivieso, 2015), tal y como se identificó la relación en este estudio.

La escala de diferencia de ritmo del CUMANIN, fue la que presentó mayores asociaciones con las subescalas del CUMANES. Esta relación puede deberse a que, al ser una escala que implica orden, teniendo en cuenta las nociones de rápido y lento; orientación: antes- después y la estructuración en la conciencia de movimientos, están vinculadas al lóbulo temporal que a su vez influye sobre el desarrollo y adquisición de funciones motoras, producción del lenguaje, audición, escritura y motricidad (Encalada \& Reino, 2013; Portellano, 2005; Rosselli et al., 2010).

Al respecto, estudios de conectividad cerebral han identificado cambios en redes neuronales interconectadas después de procesos de estimulación (Lewis et al., 2008). Un estudio llevado a cabo por Polanía, Nitsche y Paulus (2011), identificaron cambios funcionales luego de la estimulación de la memoria de trabajo por 6 semanas en jóvenes, 
especialmente en las redes en la corteza frontomedial y regiones frontoparietales. En este mismo estudio incluyeron un pilotaje con niños notando cambios funcionales similares a la de los jóvenes. Por su parte Schneider, Charpak, Ruíz-Peláez y Tessier (2012) buscaron identificar los efectos de un programa de "mamá canguro" sobre las redes neuronales de adolescentes que habían nacido con prematurez.

A partir de la estimulación magnética trasncraneal en la corteza motora primaria, identificaron activaciones de la red motora y callosa similar frente al grupo control nacido a término. Ambos estudios concluyen que la activación sincrónica de redes neuronales, la conectividad y vías cerebrales empleadas están influidas positivamente en redes cerebrales de encéfalos en desarrollo y que la intervención temprana mejora los procesos relacionados con habilidades cognitivas superiores. En nuestro estudio, el uso de estimulación temprana en niños podría favorecer el desempeño en actividades cognitivas superiores en ciertas áreas implicadas que con ayuda de la estimulación constante generan redes neuronales adecuadas.

Por lo tanto, la aplicación del PROCONI como programa de estimulación neuropsicológica en niños, podría estar relacionado con perfiles normales a largo plazo que, junto a factores medio ambientales y sociales, influyeron en la maduración neuropsicológica esperada para el grupo evaluado. Como refieren Baker-Henninhgam y López (2014), los programas de estimulación en estudios longitudinales son una tarea pendiente en el medio $y$, teniendo en cuenta los beneficios a largo plazo a nivel cognitivo, económico y social, deben implementarse como opción de estimulación y prevención de dificultades a nivel académico, social y emocional (Maulik \& Darmstadt, 2009; Belfield et al., 2006).

Como limitaciones, el presente estudio no contó con la participación de un grupo control para comparar las medidas a largo plazo del programa, esto debido a las recomendaciones del comité de ética que financió el proyecto de investigación de la Universidad Pedagógica y Tecnológica de Colombia, dictaminando que en caso de que se vieran resultados positivos en la aplicación del PROCONI en el grupo experimental, se debía aplicar el mismo tratamiento en el grupo control. Por lo tanto, se sugiere que en el diseño metodológico de los proyectos se incluya una fase de seguimiento a largo plazo en caso de pasar por un comité de ética y que permita poder llevar un seguimiento más específico sobre el efecto a largo plazo del programa. A su vez, es importante seguir diseñando e implementando programas de este tipo a nivel grupal, que ayuden, a su vez, a fortalecer las áreas relacionadas con la adquisición de habilidades escolares en los menores y evitar así problemáticas relacionadas al fracaso escolar.

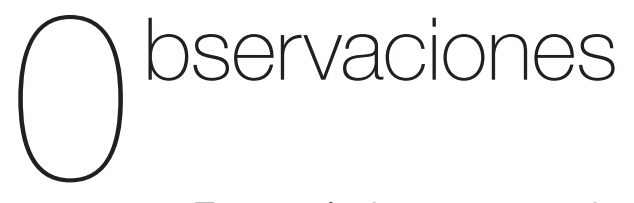

Este artículo es parte de los productos entregables del Proyecto de Investigación, código SGl 2087 aprobado por la Dirección de Investigaciones de la Universidad Pedagógica y Tecnológica de Colombia. 


\section{Referencias}

Arana, A., \& Guisely, E. (2012). Desarrollo de lenguaje comprensivo en niños de 3, 4 y 5 años de diferente nivel socioeconómico. (Tesis de Magister). Pontificia Universidad Católica de Perú, Perú.

Ardila, A., \& Rosselli, M. (2007). Neuropsicología clínica. México D.F.: Manual Moderno.

Arias, W. (2016). Ernesto Pollitt y su contribución a la psicología evolutiva en el Perú: sus investigaciones en nutrición, cognición y rendimiento escolar. Revista de Psicología PUCP 34(2), 481-500

Attanasio, O. P., Fernández, C., Fitzsimons, E. O. A., Grantham-McGregor, S. M., Meghir, C., \& Rubio-Codina, M. (2014). Using the infrastructure of a conditional cash transfer program to deliver a scalable integrated early child development program in Colombia: cluster randomized controlled trial. British Medical Journal, 349, g5785. http://doi.org/10.1136/bmj.g5785.

Ávila, A. (2012). Adaptación del cuestionario de madurez neuropsicológica de Portellano. Revista Iberoamericana de Psicología: Ciencia y Tecnología, 5(1), 91-99.

Baker-Henninhgam, L., \& López, F. (2014). Intervenciones de estimulación infantil temprana en los países en vías de desarrollo: lo que funciona, por qué y para quién. Económica, 60, 120-186.

Barnett, W. S. (1995). Long-term effects of early childhood programs on cognitive and school outcomes. The future of children, 5(3), 25-50.

Belfield, C.R., Nores, M., Barnett, W.S., \& Schweinhart, L.J. (2006). The High/Scope Perry Preschool Program: Cost-benefit analysis using data from the age-40 followup. Journal of Human Resources, 41, 162-190. doi: https://doi.org/10.3368/jhr. xli.1.162

Benavides, R., Calvache, O., Morillo, H., Ageda, A., \& Figueroa, C. (2016). Desarrollo de los trastornos de aprendizaje en el niño. Documentos de Trabajo, 2, 1-19.

Bonnier, C. (2008). Evaluation of early stimulation programs for enhancing brain development. International Journal of Paediatrics, 97(7), 853-858. http://doi. org/10.1111/j.1651-2227.2008.00834.x

Campbell, F., Ramey, C., Pungello, E., Sparling, J., \& Jhonson, S. (2010). Childhood Education: Young Adult Outcomes From the Abecedarian Project. Applied Developmental Science, 6(1), 42-57 doi: 10.1207/S1532480XADS0601_05

Campo, C., Tuesca, R., \& Campo, L. (2012). Relación entre el grado de madurez neuropsicológica infantil y el índice de talla y peso en niños de 3 a 7 años escolarizados de estratos socioeconómicos dos y tres de la ciudad de Barranquilla (Colombia). Salud Uninorte. Barranquilla, 28(1), 88-98.

Carrasco, M., Hernández, L., Roldán, J., \& Chávez, A. (2016). Desnutrición y desarrollo cognitivo en infantes de zonas rurales marginadas de México. Gaceta Sanitaria, 30(4), 304-307 doi: http://dx.doi. org/10.1016/j.gaceta.2016.01.009 
Chávez, R. (2003). Neurodesarrollo neonatal infantil. Un enfoque multi- inter y trans disciplinario de la prevención del daño. México DF, México: Editorial Médica Panamericana.

Chinome, J., Rodríguez, L., \& Parra, J. (2017). Implementación y evaluación de un programa de estimulación cognitiva en preescolares rurales. Psicología desde el Caribe 34(3), 184-203.

Chinome J., Rodríguez, \& Pineda, A. (2017). Baremación del Cuestionario de Madurez Neuropsocológica Escolar CUMANES en Boyacá. Manuscrito sin publicar.

Encalada, V., \& Reino, M. (2013). Evaluación de la Madurez Neuropsicológica de los niños y niñas de nivel inicial (Tesis de pregrado). Universidad de Cuenca, Cuenca, Ecuador.

Flores, J. \& Ostrosky, F. (2012). Desarrollo neuropsicológico de lóbulos frontales y funciones ejecutivas. México: Manual Moderno.

Galindo, G., Solovieva, Y., Machinskaya, R., \& Quintanar, L. (2016). Atención selectiva visual en el procesamiento de letras: un estudio comparativo. Revista de Estudios sobre Lectura, 15(1), 69-80. doi:10.18239/ ocnos_2016.15.1.945

Guarneros, E., \& Vega, L. (2014). Habilidades lingüísticas orales y escritas para la lectura y escritura en niños preescolares. Avances en Psicología Latinoamericana, 32(1), 2135. doi: 10.12804/apl32.1.2014.02

Gutiérrez, L., Lazarte, F., \& Alarcon, G. (2016). The importance of the neural development assessment in children aged less than 30 months in the Peruvian context. Acta Médica Peruana, 33(4), 304-308.
Knickmeyer, R. C., Gouttard, S., Kang, C., Evans, D., Wilber, K., Smith, J. K. \& Gilmore, J. H. (2008). A structural MRI study of human brain development from birth to 2 years. Journal of Neuroscience, 28(47), 12176-12182. http://doi.org/10.1523/ JNEUROSCI.3479-08.2008\r28/47/12176

Maulik, P., \& Darmstadt, G. (2009). Communitybased interventions to optimize early childhood development in low resource settings. Journal of Perinatology, 29(8), 531-542. doi: 10.1038/jp.2009.42

McKay, H., Sinisterra, L., McKay, A., Gomez, H., \& Lloreda, P. (1978). Improving Cognitive Ability in Chronically Deprived Children. Science, 200(4339), 270-278. doi: 10.1017/ CBO9781107415324.004

Medina, M., Kahn, I., Muñoz, P., Leyva, J., Moreno, J., \& Vega, S. (2015). Neurodesarrollo infantil: características normales y signos de alarma en el niño menor de cinco años. Revista Peruana de Medicina Experimental y Salud Publica, 32(3), 565-573. doi: https://doi. org/10.17843/rpmesp.2015.323.1693

Mendola, P., Selevan, S.G., Gutter, S., \& Rice, D. (2002). Factores medioambientales asociados al espectro del déficit del Neurodesarrollo. Mental Retardation and Developmental Disabilities Research Reviews, 8, 188-197.

Ministerio de Salud y Protección Social (2013). Análisis de situación de salud según regiones Colombia. Informa de la dirección de epidemiologia y demografía grupo ASIS. Recuperado de http://www. minsalud.gov.co/Documentos\%20y\%20 Publicaciones/An\%C3\%A1lisis\%20de\%20 situaci\%C3\%B3n\%20de\%20salud\%20 por\%20regiones.pdf 
Mora, R., \& Pérez, C (2013). Atención temprana comunitaria en niños con factores de riesgo de retardo del Neurodesarrollo: 1998-2008. Revista Cubana de Neurología y Neurocirugía, 3(1), 5-12.

Muñoz, M., \& Tirapu, J. (2008). Rehabilitación Neuropsicológica. Madrid, España: Editorial Síntesis.

Navarro, E., Meléndez, J., Sales, A., \& Sancerni, M. (2012). Desarrollo infantil y adolescente: trastornos mentales más frecuentes en función de la edad y el género. Psicothema, 24(3), 377-383.

Nores, M., \& Barnett, S. (2010). Benefits of early childhood interventions across the world: (Under) Investing in the very Young. Economics of Education Review, 29, 271-282. doi: https://doi.org/10.1016/j. econedurev.2009.09.001

Ortiz, P., \& Añari, M. (2013). Discrepancias en el rendimiento neuropsicológico en niños de zona rural y urbana. Revista Psicología, 3(2), 177-182.

Osorio, A., Romero, G., Bonilla, H., \& Aguado, L. (2016). Influencia del contexto socioeconómico de la comunidad sobre la desnutrición infantil en Colombia: Un enfoque multinivel para los años 2005 y 2010. Documentos de trabajo FCEA, 24, 1- 45

Papalia, E., Olds, S., \& Feldman, R. (2009). Psicología del desarrollo de la infancia a la adolescencia (11 ed.). México DF, México: Editorial McGraw Hill.

Parra, J. (2015). Evaluación de la Madurez Neuropsicológica y Programa De Corrección En Preescolares. (Tesis de pregrado). Universidad Pedagógica y Tecnológica de Colombia, Tunja, Colombia.

Parra, J., \& Rodríguez, L. (2016). Baremación del Cuestionario de Madurez Neuropsicológica Infantil CUMANIN en Boyacá para la detección temprana del fracaso escolar. Manuscrito sin publicar.

Parra, J., Rodríguez, L., \& Chinome, J. (2015). Relación entre peso al nacer y madurez neuropsicológica en preescolares de Tunja (Colombia). Pensamiento Psicológico, 13(2), 65-77. doi: 10.11144/Javerianacali. PPSI13-2.rpnm

Parra, J., Rodríguez, L., \& Chinome, J. (2016). Evaluación de la madurez neuropsicológica en preescolares. Universidad y Salud, 18 (1), 126- 137

Polanía, R., Nitsche, M. A., \& Paulus, W. (2011). Modulating functional connectivity patterns and topological functional organization of the human brain with transcranial direct current stimulation. Human brain mapping, 32(8), 1236-1249. https://doi.org/10.1002/ hbm.21104

Portellano, J. (2005). Introducción a la Neuropsicología. Madrid, España: Editorial McGraw Hill.

Portellano, J., Mateos, R., Martínez, A., Granados, J., \& Tapia, A. (2009). Cuestionario de madurez Neuropsicológica infantil CUMANIN ( $2^{\text {a }}$. ed.). Madrid, España: Editorial TEA.

Portellano, J. A., Mateos, R., \& Martínez, A. R. (2012). CUMANES. Cuestionario de Madurez Neuropsicológica Escolar. Madrid, España: Tea Ediciones. 
Ríos, J., \& Cano, I. (2016). Influencia del nacimiento prematuro en el desarrollo neuropsicológico infantil. Psicoespacios, 16, 167-192.

Rodríguez, L., Parra, J., \& Chinome, J. (2016). Implementación de un programa de corrección neuropsicológica en niños de zonas urbana y rural de Tunja (Colombia). IX Congreso Cerebro y Mente. Congreso llevado a cabo en la Universidad de Guadalajara, México

Rodríguez, M., Zapata, M., \& Puentes, P. (2008). Perfil neuropsicológico de escolares con trastornos específicos del aprendizaje de instituciones educativas de Barranquilla, Colombia. Acta Neurológica Colombiana, 24, 63-73.

Rosselli, M., Matute, E., \& Ardila, A. (2010). Neuropsicología del desarrollo infantil. México DF, México: Editorial Manual Moderno.

Sánchez, M. (2012). Perfil neuropsicológico de niños de edades comprendidas entre 4 y 7 años con antecedentes de gran prematuridad. (Tesis Doctoral). Universidad de Almería, Almería, España.

Schneider, C., Charpak, N., Ruiz-Peláez, J. G., \& Tessier, R. (2012). Cerebral motor function in very premature-at-birth adolescents: a brain stimulation exploration of kangaroo mother care effects. Acta Pediátrica, 101(10), 1045-1053 https://doi. org/10.1111/j.1651-2227.2012.02770.x
Semrud- Clikeman, M., \& Ellison, A. (2011). Neuropsicología infantil. Evaluación e intervención en los trastornos neuroevolutivos. (2. Ed.). Madrid, España: Pearson Ediciones.

Solovieva, Y., \& Quintanar, L. (2012). Formation of drawing activity in Mexican pre-school children. Psychology Research, 2(8), 479-489.

Super, C. M., Herrera, M. G., \& Mora, J. O. (1990). Long-Term Effects of Food Supplementation and Psychosocial Intervention on the Physical Growth of Colombian Infants at Risk of Malnutrition. Child Development, 61(1), 29-49. doi: http:// doi.org/10.1111/j.1467-8624.1990.tb02758.x

Urzúa, A., Ramos, M., Alday, C., \& Alquinta, A. (2009). Madurez neuropsicológica en preescolares: propiedades psicométricas del test CUMANIN. Terapia Psicológica, 28(1), 13-25. doi: http://dx.doi.org/10.4067/ S0718-48082010000100002

Valdivieso, L. (2015). Psicología cognitiva y neurociencias de la educación en el aprendizaje del lenguaje escrito y de las matemáticas. Revista de Investigación en Psicología, 17(2), 25-37. doi: https://doi. org/10.15381/rinvp.v17i2.11256

Walker, S., Chang, S., Vera-Hernández, M. \& Grantham-McGregor, S. (2011). Early Childhood Stimulation Benefits Adult Competence and Reduces Violent Behaviour. Pediatrics, 127(5), 849-857. doi:10.1542/peds.2010-2231 
pp • 85-104 Javier Humberto Parra Pulido y Lucía Carlota Rodríguez Barreto

Informes Psicológicos 